\title{
Impact of Functional Groups onto the Electronic Structure of Metal Electrodes in Molecular Junctions
}

\author{
Marc Saitner, ${ }^{\dagger}$ Felix Eberle, ${ }^{\ddagger}$ Johnny Baccus, ${ }^{\dagger}$ Marc D’Olieslaeger, ${ }^{\dagger, \S}$ Patrick Wagner, ${ }^{\dagger, \S}$ Dieter M. Kolb, ${ }^{\ddagger} \perp$
} and Hans-Gerd Boyen $*, \dagger, \S$

\author{
${ }^{\dagger}$ Institute for Materials Research (IMO), Hassselt University, B-3590 Diepenbeek, Belgium \\ ${ }^{\ddagger}$ Institute of Electrochemistry, Ulm University, D-89069 Ulm, Germany \\ ${ }^{\S}$ Division IMOMEC, IMEC, Wetenschapspark 1, B-3590 Diepenbeek, Belgium
}

\section{Supporting Information}

ABSTRACT: A better understanding of the interactions between metal electrodes and organic molecules still represents one of the key problems in molecular electronics that needs be solved in order to optimize electron transport through a molecular device. In this contribution, the impact of widely used "alligator clips" ( $\mathrm{Au}-$ thiol, $\mathrm{Pd}$-pyridine) onto the electronic structure of the respective electrodes in metalmolecule-metal junctions has been studied using photoelectron spectroscopic tools. Different electronic properties are observed for molecules with different lengths but identical terminal groups, thereby elucidating a new aspect in the complex behavior of metal/molecule contacts.

\section{INTRODUCTION}

The concept of functional groups represents one of the pillars of organic chemistry since it allows to categorize organic compounds into specific families with predictable functional properties. The knowledge about the chemistry of few generic molecular moduls gives easy access to the chemical behavior of thousands of complex organic materials, thereby assuming that the chemical reactivity of the individual module is independent of the molecular backbone where it is attached to. This specific concept is also widely used in nanoelectronics based on small organic molecules ("molecular electronics") which seeks to replace the building blocks of traditional semiconductor devices by molecular entities in order to reduce typical feature sizes by up to 2 orders of magnitude. ${ }^{1-14}$ The extraordinary success of organic molecules as new building blocks in nanoelectronics is largely based on the availability of molecular precursors with adjustable electronic properties which can be controlled by incorporating specific functional groups into the molecular design. Functional terminal groups like, e.g., thiol or pyridine are frequently exploited as "alligator clips" to firmly attach molecules to their respective metal electrodes. Such an attachment, however, is known to play a crucial role in determining the flow of electrons across a metal-moleculemetal junction. ${ }^{15-20}$ Modifications of molecular energy levels are expected as a result of chemical bonding between the terminal groups and the metal electrodes. ${ }^{19,21-23}$ On the other hand, such bonds might also affect the electronic properties of the electrodes close to the metal-molecule interfaces as has been discussed earlier. ${ }^{17,21,22,24}$

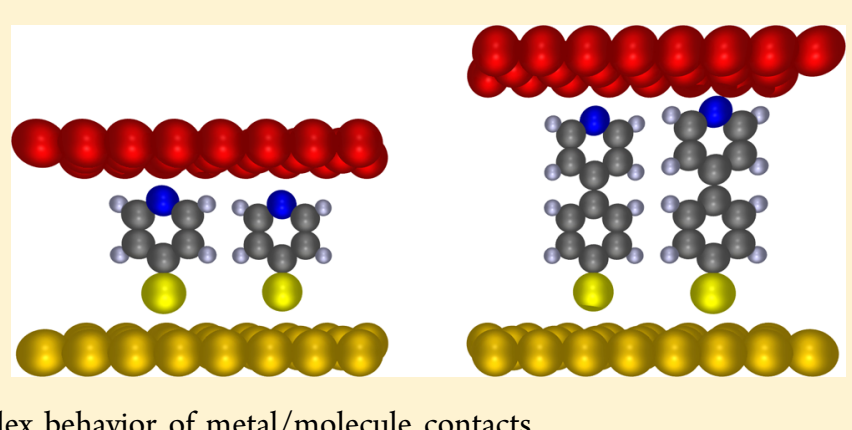

In this contribution, the impact of "alligator clips" onto the electronic structure of the metal electrodes will be studied for SAMs differing in length of the involved molecules while preserving the same terminal groups (thiol at one side, pyridine at the other side). Within a simplified picture, the use of identical terminal groups within a molecular family ${ }^{25,26}$ should have the same impact onto the metals independent of the molecular backbone. In contrast, clear modifications in the electronic structure at the metal/molecule interfaces are observed for the different molecules as will be demonstrated below.

\section{EXPERIMENTAL SECTION}

Sample Preparation. The $\mathrm{Au}(111)$ single crystals (Mateck $\mathrm{GmbH}$, Jülich, Germany) used for cyclic voltammetry, STM experiments, and XPS/UPS measurements were discs of either 4 or $12 \mathrm{~mm}$ diameter. All crystals were annealed in a hydrogen flame prior to the deposition of the organic layer. While the deposition of 4,4-mercaptopyridine (named 4-MP hereafter) SAMs and their metallization with a full Pd monolayer has been described in detail earlier, ${ }^{27}$ the procedure for the deposition of the 4-(4-mercaptophenyl)-pyridine (named 4-MPP hereafter) SAMs and their overgrowth with a full Pd monolayer essentially followed four steps:

(a) The freshly annealed $\mathrm{Au}(111)$ electrode was immersed for $2 \mathrm{~h}$ without potential control into a solution of $1 \mu \mathrm{M}$

Received: June 17, 2012

Revised: September 18, 2012

Published: September 21, 2012 
4-MPP made of ethanol (Merck, p.a.), which was deaerated to prevent 4-MPP oxidation. This resulted in the formation of a densely packed 4-MPP SAM on the $\mathrm{Au}(111)$ surface.

(b) After thoroughly rinsing the 4-MPP-modified gold electrode with ethanol and ultrapure water (Milli-Q, toc $<3 \mathrm{ppb}, 18.2 \mathrm{M} \Omega \cdot \mathrm{cm}$ ), the sample was immersed without potential control for $5 \mathrm{~min}$ in an aqueous solution of $1 \mathrm{mM} \mathrm{PdSO}_{4}$ (Alfa Aesar, 99.99\%) + 0.1 M $\mathrm{H}_{2} \mathrm{SO}_{4}$ (Merck, suprapur) to allow Pd ion adsorption/ complexation with the SAM.

(c) After rinsing again with water, the emersed electrode was then transferred to the electrochemical cell, containing $0.1 \mathrm{M} \mathrm{H}_{2} \mathrm{SO}_{4}$ only. The electrode was contacted with the electrolyte at $+0.4 \mathrm{~V}$ to prevent the unintentional reduction of adsorbed Pd(II). The potential scan started in negative direction for the electroreduction of the metal ions. All potentials are quoted vs SCE (saturated calomel electrode), although for the STM experiments a Pt wire was used as pseudoreference electrode $\left(E_{\mathrm{Pt}}=+0.55 \pm\right.$ $0.05 \mathrm{~V}$ vs SCE). Details about the experimental setups for CV and STM are described elsewhere. ${ }^{28}$

(d) As the electrochemical cycle of complexation/reduction mentioned before only yields a Pd coverage of about $1 / 3$, the cycle has been repeated 2 more times in order to achieve a full $\mathrm{Pd}$ monolayer (see the Supporting Information).

Photoelectron Spectroscopy. All photoemission spectra were measured using a commercial Physical Electronics PHI 5600LS photoemission system. Core-level spectra were acquired by X-ray photoelectron spectroscopy (XPS) using $\mathrm{Al}$ $\mathrm{K} \alpha \mathrm{X}$-rays $(1486.60 \mathrm{eV})$ for the analysis of both the chemical state of the samples and the layer sequence of the junction. The spot size of the beam was about $1 \mathrm{~mm}^{2}$, and the overall energy resolution (photons and photoelectrons) was adjusted to $0.3-$ $0.4 \mathrm{eV}$ (fwhm). Radiation damage arising from secondary electrons ${ }^{29}$ could be avoided by using a monochromator system. During the probing times, no measurable changes of the corresponding core-level intensities, or their line shapes, could be detected. The electronic structure of the Pd overlayer as well as the $\mathrm{Au}(111) / \mathrm{SAM}$ interface was characterized by ultraviolet photoelectron spectroscopy (UPS) using nonpolarized $\mathrm{He}$ II radiation $(40.8 \mathrm{eV})$ as provided by a commercial gas discharge lamp (SPECS). The binding energy scale was calibrated by means of an independent $\mathrm{Au}$ reference sample, setting the $\mathrm{Au} 4 \mathrm{f}_{7 / 2}$ core level position to $84.00 \mathrm{eV}$.

\section{RESULTS AND DISCUSSION}

Interface effects are studied by means of 4-MP as well as 4-MPP based SAMs both sandwiched between a $\mathrm{Au}(111)$ base electrode and a full $\mathrm{Pd}$ overlayer of monatomic height as sketched in Figure 1. The 4-MPP molecules are members of the larger group of biphenyl-based molecular units which lately attracted tremendous interest since they allow to tailor their electronic properties by means of the torsion angle between the two rings. ${ }^{26,30-37}$ In order to compare how the metal electrodes are affected by the attachment of the two different types of molecules, macroscopic metal/molecule/metal junctions need to be prepared and analyzed with respect to their layer sequence, the chemical state of the constituents, and, especially, their electronic structure. Since the sequential preparation of macroscopic metal/molecule/metal junctions without destroy-

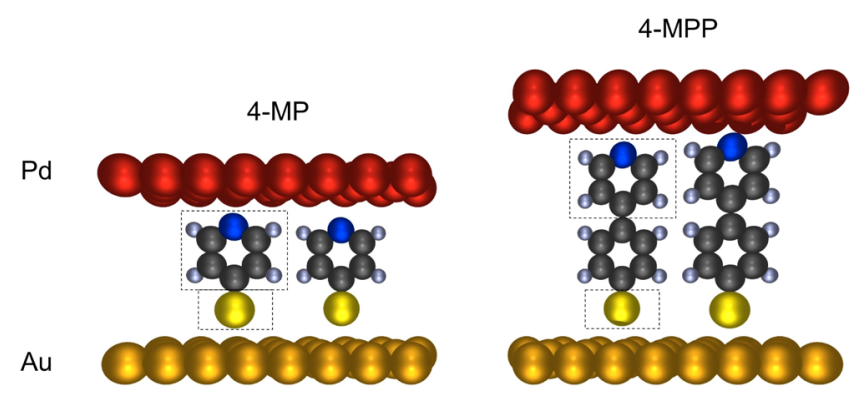

Figure 1. Schematic representation of the two different types of metal/ molecule/metal hybrid structures studied here, comprising a $\mathrm{Au}$ substrate, an organic monolayer (4-MP or 4-MPP), and a Pd overlayer (color code for the molecules: S: yellow; C: dark gray; $\mathrm{H}$ : light gray; $\mathrm{N}$ : blue).

ing the assembly by interdiffusing species still represents a challenging task, ${ }^{18}$ we relied on a two-step electrochemical method which proved to be very successful in the past. ${ }^{24,38-41}$ It is worth to mention that an interesting modification of this technique has been reported recently, ${ }^{42}$ resulting in SAMs metallized by spherical nanoparticles in contrast to flat monolayers with monatomic height as in our case.

The preparation of a 4-MP SAM on top of $\mathrm{Au}(111)$ and its successful metallization by a full monolayer of $\mathrm{Pd}$ atoms have been reported earlier. ${ }^{27}$ Consequently, for this study, it needs to be demonstrated that similar metal/molecule/metal junctions based on the longer 4-MPP molecules can be prepared as well. For that purpose, cyclic voltammetry $(\mathrm{CV})$, in-situ scanning tunneling microscopy (STM), and angle-resolved photoelectron spectroscopy (XPS, UPS) experiments were applied as complementary tools to analyze the sequential deposition of $\mathrm{Au} / 4-\mathrm{MPP} / \mathrm{Pd}$ junctions. As described in detail in the Supporting Information, a molecular SAM comprising 4-MPP molecules can indeed be prepared on top of a $\mathrm{Au}(111)$ single crystal and metallized by a (nearly full) monolayer of Pd atoms by performing the electrochemical cycle of complexation/ reduction a total of three times. This opens the door to compare the impact of two widely used "alligator clips" ( $\mathrm{Au}-\mathrm{S}$, $\mathrm{Pd}-\mathrm{N}$ ) onto the electronic structure of the respective metal electrodes for different lengths of the involved molecules (Figure 1).

In order to unravel the electronic structure of the topmost $\mathrm{Au}$ atomic layer being in direct contact with the thiolate terminal groups, angle-resolved UPS measurements were carried out on $\mathrm{Au}(111)$ crystals covered with either 4-MP (Figure 2A) or 4-MPP (Figure 2B) SAMs. Additionally, spectra were also acquired under identical experimental conditions on the pure molecules deposited as thin layers on top of Si wafers and on a clean Au single crystal all serving as reference systems for comparison. Starting with the Au reference measured in normal direction (Figure 2A), a photoelectron intensity is observed which clearly increases toward the Fermi level $E_{\mathrm{F}}$ (binding energy $E_{\mathrm{B}}=0$ ). This increase is slightly less pronounced in case of a Au substrate covered with 4-MP molecules. When changing the detection angle from normal direction to $60^{\circ}$ (thereby reducing the information depth by a factor of 2), a clear decrease in the density of states (DOS) in the vicinity of $E_{\mathrm{F}}$ is observed, resulting in a plateau-like band shape. The pure 4-MP molecules, on the other hand, are characterized by a vanishing photoelectron intensity within the depicted binding energy range, thereby confirming their semiconducting behavior. Consequently, photoelectrons emit- 

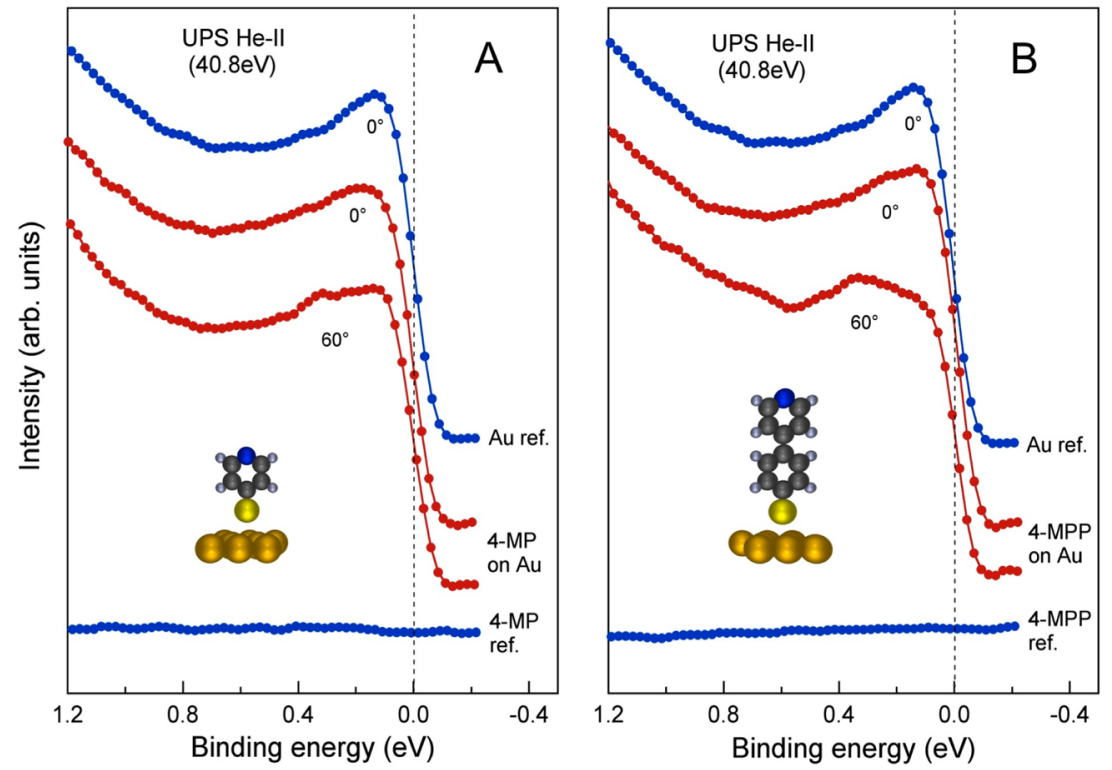

Figure 2. (A) UPS He II valence-band spectra in the low binding energy range acquired from a 4-MP covered Au surface at different detection angles. Spectra taken on a pure Au substrate and a thin 4-MP film deposited on a Si wafer are added for comparison. (B) similar sequence of spectra as presented in (A), but for a 4-MPP SAM on a Au substrate.

ted from the $\mathrm{Au}-\mathrm{SAM}$ sample can, to first approximation, be assigned to originate from the Au support alone which allows us to interpret angle-resolved spectra within this binding-energy range as representation of the surface region of the Au crystal.

Turning our attention now toward the impact of 4-MPP SAMs onto the DOS of a $\mathrm{Au}(111)$ surface (Figure 2B), different characteristics can be recognized: while, for an emission angle of $60^{\circ}$, 4-MP covered Au reveals a plateau-like DOS near $E_{\mathrm{F}}$, for the longer 4-MPP molecules, the $\mathrm{Au}-\mathrm{S}$ bond formation clearly induces a negative slope in the photoelectron distribution toward the Fermi level, thus pointing toward a stronger reduction of the density of states at $E_{\mathrm{F}}$ in the latter case. Like before, the pure molecules do not contribute to the photoelectron intensity at lower binding energies, thereby indicating that 4-MPP molecules behave semiconducting as well. These findings, on one hand, confirm qualitatively the reduction in "metallicity" of the Au surface atoms induced by chemical interaction with terminal groups as observed earlier. ${ }^{17}$ On the other hand, this reduction is found to be significantly more pronounced for 4-MPP as compared to 4-MP molecules, which contrasts the expectation of an identical impact onto the metal electrodes induced by identical (thiol) head groups of the involved molecules.

In order to derive a more quantitative picture about this phenomenon, the thermally induced broadening of the Fermi edge as well as the finite energy resolution of the electron energy analyzer has to be taken into account. This way, the decrease in intensity at $E_{\mathrm{F}}$ can be estimated for the different metal/molecule interfaces by extrapolating the respective photoelectron distributions toward the Fermi level (Figure 3, solid lines). Assuming now the topmost layer of $\mathrm{Au}$ atoms to be affected the most because of their participation in forming chemical bonds with the thiolate groups and approximating the second $\mathrm{Au}$ layer to have nearly bulk properties (which is reasonable due to the very short Thomas-Fermi screening length observed in free-electron metals like $\mathrm{Au}$ ), the measured intensity at $E_{\mathrm{F}}$ can be interpreted as superposition of the intensity arising from bulk-like (buried) $\mathrm{Au}$ and the intensity

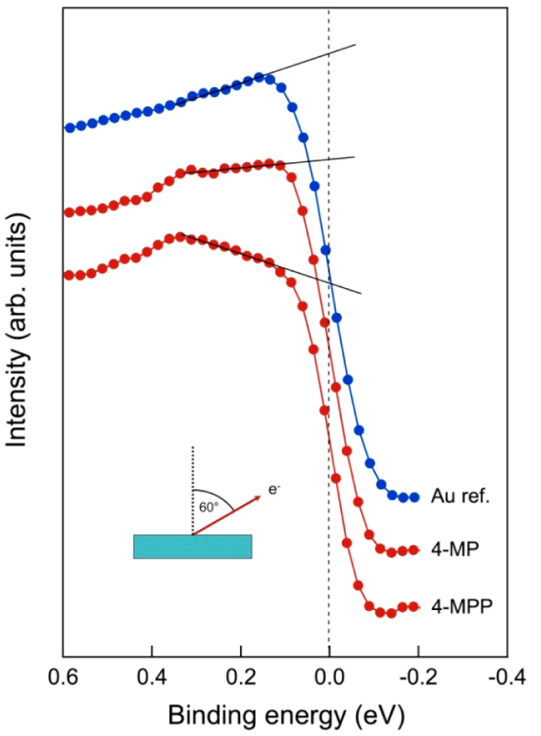

Figure 3. UPS He II spectra acquired under a detection angle of $60^{\circ}$ with respect to the surface normal for both molecular systems in comparison with a pure Au reference sample.

emitted from the top layer which is affected by chemical interactions with the different molecules. Considering a mean free path value of $0.68 \mathrm{~nm}^{43}$ for photoelectrons emitted from the Fermi level under He II irradiation and applying standard formula for the photoemission from layered structures, ${ }^{44}$ a decrease in the DOS at $E_{\mathrm{F}}$ to about half of the value of bulk $\mathrm{Au}$ can be extracted from Figure 3 in the case of 4-MP molecules. This reduction is similar to what has been reported earlier, ${ }^{17}$ thereby confirming the importance of such interface effects for 4-MP SAMs on $\mathrm{Au}(111)$.

Applying the same procedure for a Au surface covered with a 4-MPP molecular layer, the observed stronger reduction of the intensity at $E_{\mathrm{F}}$ as seen in Figure 3 is found to be equivalent to a decrease of the DOS at $E_{\mathrm{F}}$ of the interfacial metal layer by a factor of 4, which doubles the effect observed for 4-MP SAMs. 
Thus, strong evidence for distinctly dissimilar behaviors of otherwise identical terminal groups is obtained which points toward the realization of different types of "alligator clips" upon chemical attachment to the Au substrate. This clearly contrasts the expectation of identical "alligator clips" expected within the simplified picture of functional groups.

These results immediately raise the question of whether or not the other metal/molecule interface present in our molecular junctions might be affected by the formation of different "alligator clips" as well. In this case, the pyridine terminal groups of the two different molecular SAMs are used to chemically bind a Pd overlayer. The DOS of these metal overlayers can experimentally be accessed by subtracting the valence band contribution arising from their supports ( $\mathrm{Au} /$ $\mathrm{SAM}$ ) from the spectra measured on the $\mathrm{Au} / \mathrm{SAM} / \mathrm{Pd}$ hybrid systems. The corresponding results are summarized in Figure $4 \mathrm{~A}$, which depicts the photoelectron distribution curves of the
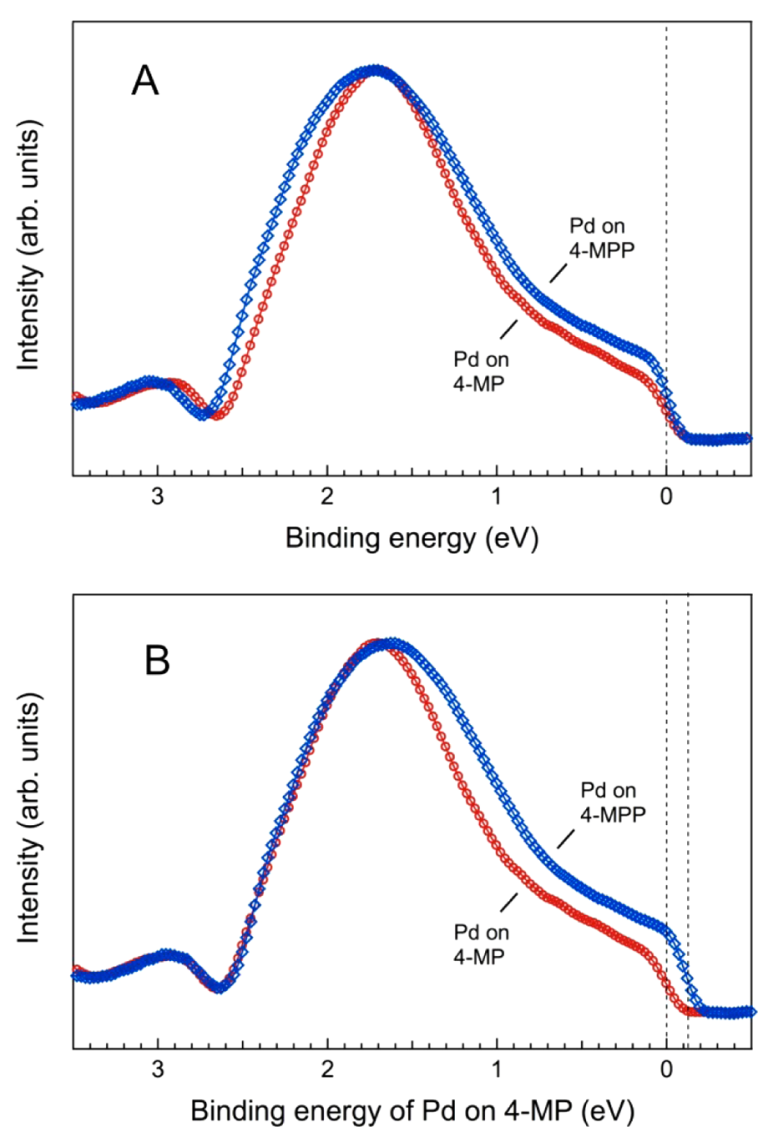

Figure 4. (A) UPS He II valence-band spectra representing the density of states of a nearly full Pd overlayer deposited on either a 4-MP (red symbols) or a 4-MPP SAM (blue symbols), respectively; for better comparison, both spectra have been normalized to the same height. (B) Same data as presented in (A), but shifted horizontally with respect to each other to line up the characteristic spectral feature observed at higher binding energy.

two Pd monolayers normalized to the same height for better comparison. In both cases, two characteristic spectral features are visible at binding energies of 1.70 and $2.90 \mathrm{eV}$ (4-MP) and 1.75 and $3.05 \mathrm{eV}$ (4-MPP), respectively. Additionaly, both overlayer systems reveal a metallic behavior which can be concluded from the presence of a step-like intensity at $E_{\mathrm{F}}$ which, again, is smeared out due to the Fermi distribution function and the finite energy resolution of the electron spectrometer. In order to better judge the specific similarities and differences between the two photoelectron distribution curves, both spectra have been shifted horizontally with respect to each other to line up the spectral feature observed at higher binding energy in Figure 4A. This way, nearly identical band shapes can be recognized for that spectral feature. On the other hand, for the band closer to the Fermi level, a larger bandwidth can be detected for a Pd monolayer on top of 4-MPP SAM. Thus, a direct comparison between the electronic structures of both Pd overlayers points toward the realization of different types of "alligator clips" for this metal/molecule interface as well.

The larger bandwidth found for Pd on 4-MPP might be discussed as consequence of line broadening resulting from a reduced lifetime of the photoionized state after emission of a photoelectron from the Pd monolayer. Typically, a photohole will be neutralized on the femtosecond scale by an electron tunneling from the $\mathrm{Au}$ substrate through the SAM into the metal overlayer. Within such a picture, tunneling through 4MPP molecules would be less efficient than tunneling through 4-MP ones due to their enlarged length. Hence, hole states in the Pd overlayer should live longer for 4-MPP as compared to 4-MP molecular layers, resulting in a reduced line broadening in the first case in contrast to our experimental results. In addition, a very different behavior in lifetime broadening should also be tracable for the small intensity maximum visible at a binding energy of about $3 \mathrm{eV}$. Here, however, nearly identical band (peak) widths can be recognized in Figure 4B. Consequently, final state effects like lifetime-induced broadening can be excluded as reason for the observed difference in bandwidth. Rather, initial-state effects like the specific details of chemical bond formation might be responsible. The smaller bandwidth in case of 4-MP molecules might reflect a decreased density of delocalized electrons in the conduction band of the Pd overlayer induced by stronger chemical bonds which tend to localize electronic states to the respective bonding partners.

Even though the chemical bonding of the different molecules toward their respective metal electrodes is formally identical $(\mathrm{Au}-\mathrm{S}, \mathrm{Pd}-\mathrm{N})$, the SAM-metal interfaces need not to be. As studies in the past few years have shown, the SAM-Au interface is quite complex involving, e.g., the presence of $\mathrm{Au}$ adatoms. ${ }^{45,46}$ Furthermore, the interfacial electronic structure is likely to depend on the film structure, i.e., the packing and orientation of the molecules. The impact of packing onto the interface energetics has been studied recently from a theoretical point of view. ${ }^{47}$ In that work, the surface coverage by molecular units was identified as one of the crucial parameters influencing electronic states at metal/molecule interfaces. Experimentally, isolated 4-MP molecules on $\mathrm{Au}$ substrates were found to leave the electronic structure of the topmost Au layers basically unaffected, $^{48}$ while dense 4-MP SAMS clearly induce a reduction in DOS at the Fermi level as found earlier ${ }^{17}$ and in the present work. The different behavior observed here for 4MP and 4-MPP SAMs might thus arise from significant differences in surface coverage for the respective molecules. However, this can be ruled out in our experiments as core-level spectroscopy revealed nearly identical $\mathrm{S}-2 \mathrm{~s} / \mathrm{Au}-4 \mathrm{~d}$ ratios for both types of molecular layers. Furthermore, no evidence could be found for a significant amount of oxidized sulfur species (see Supporting Information) which could induce fluctuations in chemical bonding at the Au/SAM interface.

The observed behavior could also be influenced by dissimilar molecular dipolar moments. This seems to be unlikely, since a 
simple redistribution of charges induced by the involved electrical fields should shift the energy levels in both metals in a rigid manner, thereby leaving the shape of the respective densities of states unaffected ("rigid band behavior"). This clearly contrasts our finding of distinct variations in band shape for the involved metals when changing the type of molecule. Furthermore, it might be speculated that dissimilarities in orientation might be the reason for the observed behavior. This, again, would point toward differences in chemical bonding to the respective metals in contrast to what is expected for identical terminal groups.

Finally, another explanation could be related to the presence of adsorbates like water molecules or atomic hydrogen which might have been deposited on top of the Pd overlayer during the electrochemical procedures. ${ }^{49,50} \mathrm{~A}$ small trace of oxygen could indeed be detected by core-level spectroscopy after metallization of the SAMs (which, however, did not result in the oxidation of the $\mathrm{Pd}$ monolayer), thus indicating the presence of water molecules at the sample surface. In addition, the adsorption of hydrogen atoms at hollow sites during the electrochemical preparation of the Pd overlayer ${ }^{49,50}$ can not be ruled out experimentally, as hydrogen is the only element which can not be identified and quantified by XPS.

A deeper understanding of the observed phenomena in general and the asymmetric behavior at both interfaces in particluar is certainly not possible without a full quantum mechanical treatment including all contributing parts of the metal/molecule/metal hybrid systems including possible adsorbates like water or hydrogen. However, the present results might help to further improve our understanding about the complex behavior of functional terminal groups used as alligator clips in planar metal/molecule/metal junctions which is an important step toward the realization of nanoelectronics based on organic molecules.

\section{CONCLUSION}

In this work, the impact of widely used alligator clips $(\mathrm{Au}-$ thiol, Pd-pyridine) onto the electronic structure of the electrodes in metal $/ \mathrm{molecule} / \mathrm{metal}$ junctions has been studied by photoelectron spectroscopic tools. Different electronic properties in the vicinity of the Fermi level are detected for molecules with different lengths but identical terminal groups (4-MP, 4-MPP). This contrasts the expectation of identical clips as anticipated from the simplified concept of functional (terminal) groups. Our experiments might help to improve the current understanding of the complex behavior of terminal groups in molecular electronics.

\section{ASSOCIATED CONTENT}

\section{S Supporting Information}

Cyclic voltammetry, in-situ scanning tunneling microscopy as well as (angle-resolved) X-ray photoelectron spectroscopy results demonstrating the successful preparation of Au/4-MPP/ $\mathrm{Pd}$ junctions. This material is available free of charge via the Internet at http://pubs.acs.org.

\section{AUTHOR INFORMATION}

\section{Corresponding Author}

*E-mail: hansgerd.boyen@uhasselt.be.

\section{Notes}

The authors declare no competing financial interest.

\section{ACKNOWLEDGMENTS}

We thank Dr. G. Götz, Institute of Organic Chemistry II, University of Ulm, for the synthesis of the 4-MPP molecular precursors. Financial support by the Flemish Science Foundation (FWO) within the Odysseus program, the Methusalem project "NANO", and the German National Science Foundation (DFG) within SFB 569 is gratefully acknowledged.

\section{DEDICATION}

${ }^{\perp}$ In memory of an outstanding scientist and person.

\section{REFERENCES}

(1) Aviram, A.; Ratner, M. A. Chem. Phys. Lett. 1974, 29, 277-283.

(2) Introducing Molecular Electronics; Cuniberti, G., Fagas, G., Richter, K., Eds.; Springer: Berlin, 2005.

(3) Cuevas, J. C.; Scheer, E. Molecular Electronics: An Introduction to Theory and Experiment; World Scientific Pub Co.: Singapore, 2010.

(4) Reed, M. A.; Zhou, C.; Muller, C. J.; Burgin, T. P.; Tour, J. M. Science 1997, 278, 252-254.

(5) Seminario, J. M.; Tour, J. M. Ann. N. Y. Acad. Sci. 1998, 852, 6894.

(6) Fink, H.-W.; Schönenberger, C. Nature 1999, 398, 407-410.

(7) Chen, J.; Reed, M. A.; Rawlett, A. M.; Tour, J. M. Science 1999, 286, 1550-1552.

(8) Joachim, C.; Gimzewski, J. K.; Aviram, A. Nature 2000, 408, $541-548$.

(9) Gittins, D. I.; Bethell, D.; Schiffrin, D. J.; Nichols, R. J. Nature 2000, 408, 67-69.

(10) Porath, D.; Bezryadin, A.; Vries, S. d.; Dekker, C. Nature 2000, 403, 635-638.

(11) Reichert, J.; Ochs, R.; Beckmann, D.; Weber, H. B.; Mayor, M.; Löhneysen, H. v. Phys. Rev. Lett. 2002, 88, 176804.

(12) Seminario, J. M. Nat. Mater. 2005, 4, 111-113.

(13) Rocha, A. R.; Garcia-Suarez, V. M.; Bailey, S. W.; Lambert, C. J.; ferrer, J.; Sanvito, S. Nat. Mater. 2005, 4, 335-339.

(14) Blum, A. S.; Kushmerick, J. G.; Long, D. P.; Patterson, C. H.; Yang, J. C.; Henderson, J. C.; Yao, Y.; Tour, J. M.; Shashidhar, R.; Ratna, B. Nat. Mater. 2005, 4, 167-172.

(15) Hipps, K. W. Science 2001, 294, 536-537.

(16) Kushmerick, J. G. Mater. Today 2005, July/August 2005, 26-30.

(17) Boyen, H.-G.; Ziemann, P.; Wiedwald, U.; Ivanova, V.; Kolb, D. M.; Sakong, S.; Gross, A.; Romanyuk, A.; Büttner, M.; Oelhafen, P. Nat. Mater. 2006, 5, 394-399.

(18) Haick, H.; Cahen, D. Prog. Surf. Sci. 2008, 83, 217-261.

(19) Zotti, L. A.; Kirchner, T.; Cuevas, J. C.; Pauly, F.; Huhn, T.; Scheer, E.; Erbe, A. Small 2010, 6, 1529-1535.

(20) Kim, Y.; Hellmuth, T. J.; Burkle, M.; Pauly, F.; Scheer, E. ACS Nano 2011, 5, 4104-4111.

(21) Sun, Q; Selloni, A.; Scoles, G. J. Phys. Chem. B 2006, 110, 3493-3498.

(22) Wang, J. G.; Selloni, A. J. Phys. Chem. A 2007, 111, 1238112385.

(23) Heimel, G.; Rissner, F.; Zojer, E. Adv. Mater. 2010, 22, 24942513.

(24) Manolova, M.; Boyen, H. G.; Kucera, J.; Gross, A.; Romanyuk, A.; Oelhafen, P.; Ivanova, V.; Kolb, D. M. Adv. Mater. 2009, 21, 320324.

(25) Hybertsen, M. S.; Venkataraman, L.; Klare, J. E.; Whalley, A. C.; Steigerwald, M. L.; Nuckolls, C. J. Phys.: Condens. Matter 2008, 20, 374115 .

(26) Mishchenko, A.; Vonlanthen, D.; Meded, V.; Bükle, M.; Chen, L.; Pobelov, I. V.; Bagrets, A.; Viljas, J. K.; Pauly, F.; Evers, F.; Mayor, M.; Wandlowski, T. Nano Lett. 2010, 10, 156-163.

(27) Eberle, F.; Saitner, M.; Boyen, H.-G.; Kucera, J.; Gross, A.; Romanyuk, A.; Oelhafen, P.; D’Olieslaeger, M.; Manolova, M.; Kolb, D. M. Angew. Chem., Int. Ed. 2010, 49, 341-345. 
(28) Ivanova, V.; Baunach, T.; Kolb, D. M. Electrochim. Acta 2005, $50,4283-4288$.

(29) Jäger, B.; Schürmann, H.; Müller, H. U.; Himmel, H.-J.; Neumann, M.; Grunze, M.; Wöll, C. Z. Phys. Chem. 1997, 202, 263272.

(30) Venkataraman, L.; Klare, J. E.; Nuckolls, C.; Hybersten, M. S.; Steigerwald, M. L. Nature 2006, 442, 904-907.

(31) Finch, C. M.; Sirichantaropass, S.; Bailey, S. W.; Grace, I. M.; Garcia-Sanchez, V. M.; Lambert, C. J. J. Phys.: Condens. Matter 2008, 20, 022203.

(32) Pauly, F.; Viljas, J. K.; Cuevas, J. C.; Schön, G. Phys. Rev. B 2008, $77,155312$.

(33) Xia, C.-J.; Fang, C.-F.; Zhao, P.; Xie, S.-J.; Liu, D.-S. Phys. Lett. A 2009, 373, 3787-3794.

(34) Kondo, H.; Nara, J.; Kino, H.; Ohno, T. J. Phys.: Condens. Matter 2009, 21, 064220.

(35) Busiakiewicz, A.; Karthäuser, S.; Homberger, M.; Kowalzik, P.; Waser, R.; Simon, U. Phys. Chem. Chem. Phys. 2010, 12, 10518-10524.

(36) Brito Silva, C. A.; da Silva, S. J. S.; Granhen, E. R.; Leal, J. F. P.;

Del Nero, J.; Pinheiro, F. A. Phys. Rev. B 2010, 82, 085402.

(37) Bürkle, M.; Viljas, J. K.; Vonlanthen, D.; Mishchenko, A.; Schön, G.; Mayor, M.; Wandlowski, T.; Pauly, F. Phys. Rev. B 2012, 85, 075417.

(38) Baunach, T.; Ivanova, V.; Kolb, D. M.; Boyen, H.-G.; Ziemann, P.; Büttner, M.; Oelhafen, P. Adv. Mater. 2004, 16, 2024-2028.

(39) Manolova, M.; Ivanova, V.; Kolb, D. M.; Boyen, H.-G.; Ziemann, P.; Büttner, M.; Romanyuk, A.; Oelhafen, P. Surf. Sci. 2005, 590, 146-153.

(40) Manolova, M.; Kayser, M.; Kolb, D. M.; Boyen, H.-G.; Ziemann, P.; Mayer, D.; Wirth, A. Electrochim. Acta 2007, 52, 2740-2745.

(41) Eberle, F.; Kayser, M.; Kolb, D. M.; Saitner, M.; Boyen, H.-G.; D’Olieslaeger, M.; Mayer, D.; Wirth, A. Langmuir 2010, 26, 47384742.

(42) Silien, C.; Lahaye, D.; Caffio, M.; Schaub, R.; Champness, N. R.; Buck, M. Langmuir 2011, 2567-2574.

(43) Penn, D. R. Phys. Rev. B 1981, 35, 482-486.

(44) Lin, T.-S.; Partin, W. J.; Damminga, G. M.; Parrill, T. M.; Lee, W.-J.; Chung, Y.-W. Surf. Sci. 1987, 183, 113-122.

(45) Woodruff, D. P. Phys. Chem. Chem. Phys. 2008, 10, 7211-7221.

(46) Hakkinen, H. Nat. Chem. 2012, 4, 443-455.

(47) Romaner, L.; Heimel, G.; Zojer, E. Phys. Rev. B 2008, 77, 045113.

(48) Koslowski, B.; Tschetschetkin, A.; Maurer, N.; Ziemann, P. Phys. Chem. Chem. Phys. 2011, 13, 4045-4050.

(49) Kucera, J.; Gross, A. Beilstein J. Nanotechnol. 2011, 2, 384-393.

(50) Kucera, J.; Gross, A. Phys. Chem. Chem. Phys. 2012, 14, 23532361. 